\title{
Directives sur le tri des patients aux soins intensifs
}

\section{Michelle Salathé}

lic. iur, MAE, responsable du ressort Ethique et Secrétaire générale adjointe de I'ASSM

Compte tenu de la vitesse à laquelle le virus Covid-19 (SARS-CoV-2) se propage, nous sommes confrontés à une situation exceptionnelle avec un afflux massif de patientes et de patients dans les hôpitaux de soins aigus. En cas de pénurie de ressources, des décisions de rationnement sont incontournables. L’Académie Suisse des Sciences Médicales (ASSM) et la Société suisse de médecine intensive (SSMI) ont publié des directives communes portant sur les décisions de rationnement dans les unités de soins intensifs.

Dans une première phase, il est possible de remédier à cette situation par la limitation des interventions électives, le transfert de patients vers des unités de soins intermédiaires, l'extension des places de traitement avec des respirateurs artificiels ainsi que le renoncement aux options de traitement qui requièrent des effectifs importants. En cas de pénurie de ressources, des décisions de rationnement sont incontournables. La charge pesant sur le personnel médical est alors extrêmement élevée. Il est d'autant plus important que les mêmes critères d'admission et de maintien en soins intensifs soient appliqués dans l'ensemble de la Suisse. Publiées le 20 mars 2020, les directives définissent cette base. Lorsque les expériences pratiques et les nouvelles connaissances l'exigent, elles seront adaptées.

Ces directives complètent les directives de l'ASSM «Mesures de soins intensifs» (2013) et concernent uniquement une petite partie des patients atteints du coronavirus, à savoir le groupe des patients gravement malades ayant besoin d'un traitement intensif. Les patients Covid-19 et les autres patients qui ont besoin de soins intensifs sont traités selon les mêmes critères.

\section{Pronostic à court terme comme facteur décisif}

Selon les directives, le pronostic à court terme est le facteur décisif pour le tri des patients. Lors de l'admission aux soins intensifs, les patients dont le pronostic de sortie de l'hôpital est favorable avec une thérapie de soins intensifs, mais défavorable sans soins intensifs, ont la priorité absolue; il s'agit des patients qui pro-

\footnotetext{
fitent le plus des soins intensifs.
}

Lâge n'est pas en soi un critère à prendre en compte. Il accorderait aux personnes âgées moins de valeur qu'aux plus jeunes et, en ce sens, viole l'interdiction de discriminer inscrite dans la Constitution fédérale. Néanmoins, l'âge est indirectement pris en compte dans le cadre du critère "pronostic à court terme», car les personnes âgées souffrent plus souvent de comorbidités. Dans le contexte du Covid-19, l'âge est également un facteur de risque de mortalité qui doit être pris en compte.

Des critères supplémentaires tels que le tirage au sort, "premier arrivé, premier servi» (first come, first served), la priorité aux personnes ayant une valeur sociale élevée etc. ne doivent pas être appliqués.

\section{Recommandations pour les soins palliatifs}

L'infection par le coronavirus menace toute la population. Toutefois, les personnes âgées, qui sont souvent polymorbides et fragiles, sont les plus exposées à une évolution grave et fatale. Les maladies les plus graves avec un pronostic défavorable et les situations de fin de vie nécessitent des soins palliatifs de qualité pour les personnes concernées. La situation actuelle de pandémie et la publication des directives de l'ASSM et de la SSMI ont incité la Société de gériatrie palliative (FGPG) à élaborer des recommandations complémentaires axées sur la pratique. L'ASSM se félicite de cette démarche.

Les directives sur le tri des patients et les recommandations en matière de soins palliatifs sont publiées en allemand, français, italien et anglais sur le site web de I'ASSM: assm.ch/fr/coronavirus 